\title{
(
}

\section{Using multi-objective optimization to design parameters in electro-discharge machining by wire}

\author{
Alberto Ochoa ${ }^{\mathrm{a}}$, Lourdes Margain ${ }^{\mathrm{b}}$, Francisco Ornelas ${ }^{\mathrm{b}}$, Sandra \\ Jiménez ${ }^{\mathrm{b}}$, Teresa Padilla ${ }^{\mathrm{b}}$ \\ ${ }^{a}$ Departamento de Energía Eléctrica y Computación, Universidad Autónoma de Ciudad Juárez. \\ ${ }^{\text {b }}$ Departamento de Posgrado e Investigación,, Universidad Politécnica de Aguascalientes.
}

KEYWORD

Multi-objective optimization Genetic Algorithms

Robust Design
ABSTRACT

The following paper describes the main objective to follow the methodology used and proposed to obtain the optimal values of WEDM process operation on the machine Robofil 310 by robust parameter design (RPD) of Dr. G. Taguichi [TAGUCHI, G. 1993], through controllable factors which result in more inferences regarding the problem to noise signal ( $/ N$ ), which for this study is the variability of the hardness of samples from 6061, also studied the behaviour of the output parameters as the material removal rate (MRR) and surface roughness $(R a)$, subsequently took the RPD orthogonal array and characterized the individuals in the population, each optimal value is a gene and each possible solution is a chromosome, used multi-objective optimization using Nondominated Sorting Genetic Algorithm to cross and mutate this population to generate better results MRR and Ra.

\section{Introduction}

Wire Electric discharge machining (WDEM) metallurgical process, which erodes the material of the workpiece by a series of sparks area made between the workpiece and the wire (electrode) separated by a thin film of fluid or liquid dielectric supply. The wire cuts like a band saw, but in-stead of teeth sparks made the cut, creating a path for each download, which ionizes the fluid in the slot area rises to extremely high temperatures, so casting material is removed. The particles which are generated by removal of material are segregated in the dielectric supply and its flow through is filtered and remote from the system. It is used the machine Robofil 310 to obtain the optimal values and a Non-dominated Sorting Genetic Algorithm in order to generate better MRR and Ra results.

\section{Material and methods}

In the early experimentation man's need to control the areas that directly affect their lives routed efforts toward deeper understanding of the DOE (Design of Experiments) where they took great minds such was the case and the legacy he left Sir Ronald Fisher. He was a great researcher using the basis of statistical methods, innovated design and development experiments. The development of testing methods is strengthened along with the technologies in environments determined by the conditions and limitations of companies or countries, this leads to complex methods of experimentation.

Today modern methods of experimental design used in all areas including industrial, commercial and financial. You can understand how the experiment designed where deliberate changes are induced in the input variables of a 
process, so that may be possible to identify the causes of changes in output responses.

One of the tools is the full factorial DOE design that uses a series of experiments investigating one factor at a time, has two advantages. The first is that the factorial experiments are much more efficient to estimate the main effects and the second reason is that the interaction between the factors can be evaluated in a factorial experiment. Fractional Factorial is defined as a fraction of the full factorial design , in other words, are performed only part of the full factorial experiments, this follows from the need for companies to minimize costs of research and time that you spend on this.

Ensuring a good job that supports the quality the RPD is the part of the methodology of Taguchi [TAGUCHI, G. 1993] involving designs of fractional treatments where the choice of factors and levels of each factor are crucial aspects of the design of the experiment and will be dictated by the knowledge matter and time constraints or cost experiment.

In the terminology of Taguchi [TAGUCHI, G. 1993] all factors involved in the RPD. The types of factors in the study, beginning with the signal factor and the quality characteristic or factors often named output.

Moreover the controllable factors are called parameters, which are not controlled are called noise factors. The noise variables or factors are most sensitive to changes in environmental conditions during production and, therefore, transmits responses to the variability in the process of interest. The interaction of factors is important to determine how the findings of the experiment can be applied generally. Factor systems are often complex, especially if a number of factors to be analysed is large.

The concept of robust design experimentation identification represents the shape of the noise factors that are not controllable, considered explicitly or implicitly, where its effect is to minimize disappear or indirectly, i.e. without directly control levels factor can be controlled, to find the right combination of these factors effect of noise is minimized [KUEHL, R. 2001]. An elementary lens of robust design is the combination of levels of the controllable factors, in which no noise factors affect the process, although these are not controlled.

\section{Theory/calculation}

The methodology proposed by Dr. Taguchi [TAGUCHI, G. 1993] for robust parameter design (RPD) is a series of steps in which are contained the phases of the design of experiments, in order to strengthen the WEDM process [MAHAPATRA, S. et al. 2006] to the noise generated by the variability of toughness throughout the entire material.

In this research is analyzed unconventional WEDM process in cutting 6061 specification aluminum specimens in a machine Robofil 310. Controllable factors have been identified and noise conditions, which affects Ra documentary research and MRR. It strengthens the process by applying Taguchi [TAGUCHI, G. 1993] methodology.

In a second stage using genetic algorithms, which is characterized according to the orthogonal array of individuals RPD, population, genes and chromosomes, based on the fact that two objectives are analyzed becomes a multi-objective optimization study. Which aims to maximize the MRR and minimize Ra.

\subsection{Parameters and factors of WEDM}

The machine Robofil 310 works by technology, in other words, the ideal values assigned according to the characteristics of the material to be processed, so that when programming a new technology for 6061 is thrown ideal values, which are composed of two forms of fixed parameters remain constant throughout the experiment and the controllable parameters which research suggests three principles: Pulse ON, Pulse Off and Wire feed, which will be subsequently chromosome genes.

Another important factor is the noise, so that a material meets the specifications and rigorously including hardness, certification is required to ensure that the material actually meets what is stated in the certificate.

In the following tables (1 and 2) are observed values of each parameter and operating levels used for the RPD, and the noise factor and 
Rockwell Hardness values "B" [LIAO, Y. et al. 1997].

\begin{tabular}{lllll}
\hline Parameter & $\begin{array}{l}\text { Level } \\
1 \\
\text { (low) }\end{array}$ & $\begin{array}{l}\text { Level } \\
2 \\
\text { (high) }\end{array}$ & Optimal & Unit \\
\hline $\begin{array}{l}\text { Pulse ON- } \\
\text { time A }\end{array}$ & 0.6 & 1.2 & 1.0 & $\mu \mathrm{s}$ \\
$\begin{array}{l}\text { Pulse } \\
\begin{array}{l}\text { OFF-time } \\
\text { B }\end{array}\end{array}$ & 6.0 & 10.0 & 8.0 & $\mu \mathrm{s}$ \\
$\begin{array}{l}\text { Wire } \\
\text { speed WS }\end{array}$ & 6.0 & 12.0 & 9.0 & $\mathrm{~m}$ \\
\hline \multicolumn{3}{c}{ Table. 1. Levels of the parameters } \\
\hline
\end{tabular}

\begin{tabular}{lll}
\hline $\begin{array}{l}\text { Noise factor } \\
\text { (hardness) }\end{array}$ & Value (HRB) & $\begin{array}{l}\text { Confidence } \\
\text { Interval (HRB) }\end{array}$ \\
\hline Level 1 & 52.96 & $52.54,53.37$ \\
Level 2 & 55.21 & $54.71,55.71$ \\
\hline \multicolumn{3}{c}{ Table. 2. Levels Noise Factor }
\end{tabular}

The initial experiment established each factor in only two levels, so that significant main effects and interactions can be quickly identified and explored further.

Pulse ON-time (A). This parameter is the duration of the spark erosion rates which are primarily affected by the pulse parameters. The spark is a bridge, the current is generated and removal work is accomplished. The longer the spark is maintained, the greater the removal of material. Therefore, the resultant craters are larger and deeper, and the surface is just rougher.

Pulse Off-time (B). Against part of the pulse On-time is the duration of this pulse and resting place when ionized again the die Electric can affect the speed of operation in general, the longer the off-time is longer the time machining. But this is an integral part of the EDM process and must exist. The Off-time also regulates the stability of the process.

Wire feed speed (WS). This parameter enters the study due to the strong interaction we have with the two pulses, the parameter itself regulates the feed rate of the thread through a nozzle disposed servos.
Orthogonal Array. The orthogonal arrangement of two noise levels for Robust Parameter Design is of the following form for consideration (see table 3).

Orthogonal Array $\mathrm{L}_{4}=2^{3}$.

\begin{tabular}{|c|c|c|c|c|c|}
\hline Block & A & B & WS & Noise & Noise \\
\hline 1 & 1 & 1 & 1 & $\Gamma$ & $I$ \\
\hline 2 & 1 & 2 & 2 & $\sum$ & 宓 \\
\hline 3 & 2 & 1 & 2 & 要 & D \\
\hline 4 & 2 & 2 & 1 & D & $\bar{D}$ \\
\hline
\end{tabular}

\section{Results and Discussion}

\subsection{Output Variables MRR y Ra}

After performing the experimental results are analyzed, the following table 4 shows the data obtained from each block, taking the MRR and $\mathrm{Ra}$ as output variables or characteristics that we intend to optimize quality, we used the statistical tool Minitab 16 in order to study their behavior.

\begin{tabular}{lllll}
\hline Block & $\begin{array}{l}\text { Noise } \\
\text { Level }\end{array}$ & $\begin{array}{l}\text { MRR } \\
\left(\frac{\tilde{n}^{2}}{h r}\right)\end{array}$ & $\begin{array}{l}\text { Machine } \\
\text { Time } \\
(\text { main) }\end{array}$ & $\begin{array}{l}\text { Roughness } \\
(\mu \mathrm{in})\end{array}$ \\
\hline 1 & 1 & 3.765 & 15.26 & 124.16 \\
2 & 1 & 7.294 & 9.50 & 135.33 \\
3 & 1 & 4.308 & 13.56 & 128.66 \\
4 & 1 & 9.220 & 6.40 & 157.33 \\
5 & 2 & 3.609 & 15.42 & 124.16 \\
6 & 2 & 6.254 & 10.00 & 135.33 \\
7 & 2 & 4.072 & 14.20 & 128.66 \\
8 & 2 & 8.923 & 6.52 & 157.33 \\
\hline \multicolumn{5}{c}{ Table. 4. Results of MRR and Ra }
\end{tabular}

The behavior of the quality characteristics or output variables MRR and $\mathrm{Ra}$, is determined that seeks to maximize MRR and Ra seeks to minimize. The following table 5 shows how the type of property and signal / noise ratio for each response variable is identified. 


\begin{tabular}{lll}
$\begin{array}{l}\text { Response } \\
\text { Variable }\end{array}$ & Characteristics & $\begin{array}{l}\text { Signal/Noise } \\
(\mathrm{S} / \mathrm{N})\end{array}$ \\
\hline $\mathrm{Ra}$ & $\begin{array}{l}\text { While smaller } \\
\text { is better }\end{array}$ & $\mathbf{- 1 0} \log \left[\frac{\mathbf{1}}{n} \sum_{i=1}^{n} \boldsymbol{Y}_{\tilde{i}}^{2}\right]$ \\
MRR & $\begin{array}{l}\text { While bigger } \\
\text { is better }\end{array}$ & $\mathbf{- 1 0} \log \left[\frac{1}{n} \sum_{i=1}^{n} \frac{1}{Y_{i}^{2}}\right]$ \\
\hline
\end{tabular}

Table. 5. Noise signal

\subsection{Select Values to Strengthen}

After choosing the factors and their levels that most impacted the quality characteristics is obtained optimal values. Implementing predicted levels and performing test runs the following gains are obtained. In the case that the process needs a roughing treatment maximizing material removal rate, the expected gain in this design is $19.1501 \mathrm{~S} / \mathrm{N}$ ratio. In the case that the process need a fine finish treatment, the surface roughness is minimized by applying this robust design, and you get a gain of $41.8796 \mathrm{~S} / \mathrm{N}$ ratio. The optimal levels of operation selected for MRR are: Pulse ON-time $(A)=2$, Pulse Off-time $(B)=2$ and Wire speed $(W s)=1$. The optimal levels of operation selected for Ra are: Pulse ON-time $(\mathrm{A})=1,2$ Pulse Off-time $(\mathrm{B})=1$ and Wire speed $(\mathrm{Ws})=1$.

The research sets the parameters at two levels of experimentation, this to identify important effects that are conducted on the response variables interact to simultaneously control the three parameters, the studied further observed:

Strong interactions are taken into controlling factors are: Length of drive (click on time) "A" and wire feed speed "Ws". Time between pulses (off press time) "B" and wire feed speed "Ws".

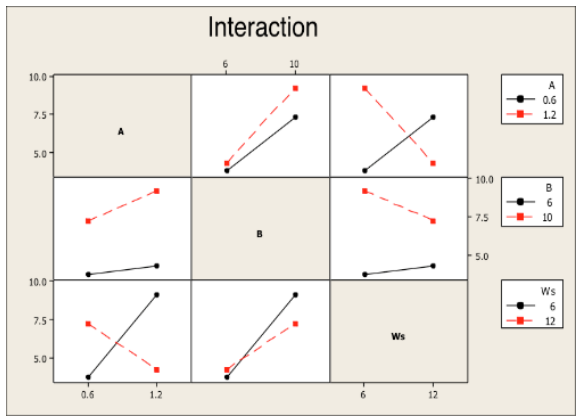

Fig. 1. Interactions for MRR.

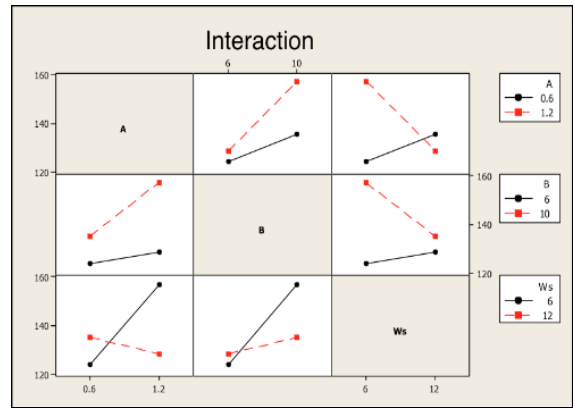

Fig. 2. Interactions for Ra.

\subsection{Genetic algorithms (GA)}

GA are based on a study of computer systems trying to understand and copy adaptation of processes and mechanisms in the evolution towards the improvement and monitoring of the effects of these processes and mechanisms. In other words, evolutionary computation algorithms are trying to exploit the properties of the fields related to population genetics. GA share similar to the early stages of DOE where adaptation through a process learns about beneficial change through trial and error are possible solutions of a population properties, the environment is defined by a specific objective function for each problem. In each case, the evolutionary process addresses the process of adaptation of the population, usually addressing the mechanisms of evolution are the cross, mutation and migration. Reproduction is accomplished by copying the best individuals from one generation to the next, the best solution will be improving from one generation to the next cyclically to comply with the proposed number of generations in the algorithm. The crosses were carried out with a rate of probability assigned. The genetic mutation takes into account the diversity in the population by random replacement of individuals in the population, and likewise that the crossing is done by a certain percentage of probability in the programming of the algorithm. In Addition migration of Individuals Between subpopulations is used if the island model is employed. Standard genetic programming algorithm is expressed as in Fig. 3. 


\begin{tabular}{|l|}
\hline The standard GA \\
\{ \\
Generate initial population. Sa \\
Assess population Sa \\
While stopping criteria not satisfied repeat \\
$\left\{\begin{array}{l}\text { Select elements of Sa Sa put }+1 \\
\text { Elements crossing in Sa Sa and put }+1 \\
\text { Mutate elements in Sa Sa and put }+1 \\
\text { Evaluate new population Sa1 } \\
\text { Sa }=\text { Sa }+1\} \\
\}\end{array}\right.$ \\
\hline \multicolumn{1}{c}{ Fig. 3 Standard genetic programming algorithm } \\
\hline
\end{tabular}

\subsection{Multi-objective optimization (MOO) for WEDM}

In this study, the MOO is a tool that quantitatively determines the relationship between quality characteristics MRR and Ra with the optimal combination of machining parameters.

Confirmation is the final step in any process of design of experiments, the purpose is to validate the conclusions drawn during the analysis in this case validate the results of the RPD. Nondominated Sorting Genetic Algorithm (NSGA) was used to confirm the optimal machining parameters for multiple output targets by using combinations proposed by the RPD, this particular case there are two objectives MRR and $\mathrm{Ra}$ where the former seeks to maximize and minimize the second, i.e. the greater the improvement in machining performance outputs in the MRR and the minimum value is the best outlet for Ra.

NSGA provides a pseudo-code listing of the classification to minimize a (MRR) and maximize another function (Ra). The two objective functions were determined by applying a linear regression on the values of the proposed orthogonal array in the RPD and the results of the output variables.

The following figure shows the equations describing the two objective functions within the code of NSGA -II, the objective "0 " refers to the MRR and aims to maximize , therefore the objective "1" is the $\mathrm{Ra}$ and seeks to minimize.

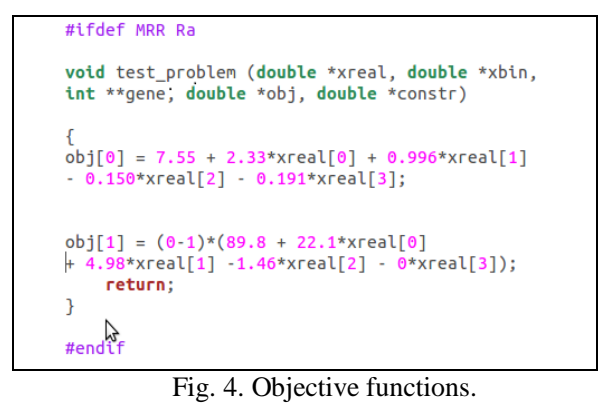

The code has been written for posix compliant operating systems and uses standard piping method provided by GNU C library. The routines should work on any unix and unix like OS having gnuplot installed and which are posix compliant.

\subsection{NSGA-II: Non-dominated Sorting Genetic Algorithm - II}

The NSGA-II is an evolutionary algorithm belong to the field of evolutionary computation inspired by the study process and mechanisms of biological evolution. The process of Darwinian evolution by natural selection that served to explain the variety of species and their adaptation to the environment. The mechanisms described how the evolution took place through the alteration, modification and reproduction of genes or genetic material [KALYANMOY, D. et al. 2002]. The objective of the NSGA-II algorithm to improve the fit is WEDM adaptive solutions of the population limited to a Pareto front by a set of objective functions in this case two objective functions, maximize and minimize Ra MRR. The algorithm uses an evolutionary process to substitute for evolutionary operators including selection, genetic crosses, and genetic mutation. The population is classified into a hierarchy of groups based on the population by Pareto dominance. Similarity between the members of each subgroup is evaluated in the Pareto front, and the resulting groups and similarity measures are used to promote a diverse of solutions. 


\subsection{Parameters of WEDM in NSGA-II}

The parameters characterizing the problem are used in the RPD, with 4 real variables, three factors control for DPR are: A, B and Ws and add the factor noise hardness of the material more as a real variable. This is each individual has 4 genes that vary in ranges previously estimated by the RPD. The range or boundaries of real variables or genes on chromosome; units they are measured in the RPD settled in this part only quantifiable value in real numbers is taken. The limits on real variables of level 1 (low) are: Pulse ON-time $A=0.6$, Pulse Off-time $B=6.0$, Wire speed WS $=6.0$ and Wire speed WS $=6.0$. The limits on real variables of level 2 (high) are: Pulse ON-time $A=1.2$, Pulse Off-time $B=10.0$, Wire speed $\mathrm{WS}=12.0$ and Wire speed WS=66.21. The population size should be in multiples of four because there are four real variables studied, being thus a population of 400 individuals, the number of generations is estimated by analyzing NSGA applications in different samples was estimated and found to optimal 100 ensures generation where the number of generations with no changes in any variable. In Figure 4, all relevant parameters are observed to characterize the problem in this case the WEDM process using NSGA.

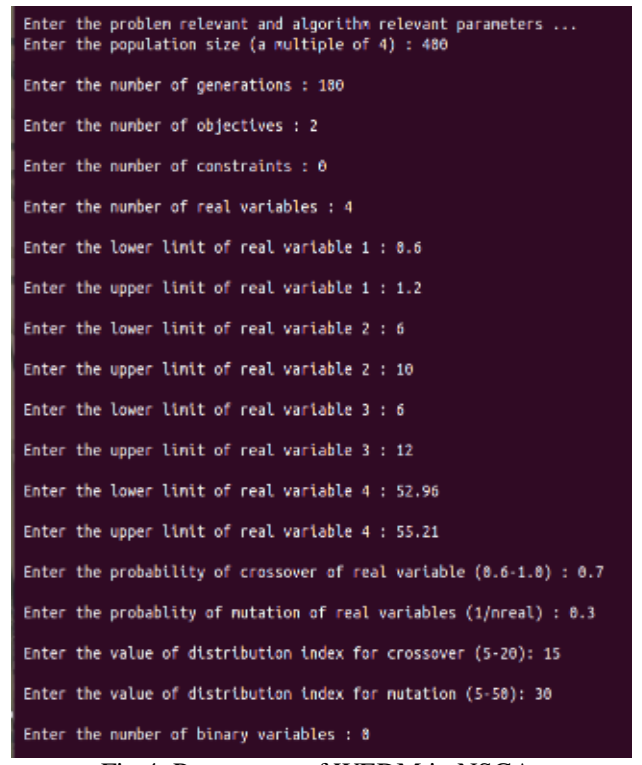

Fig.4. Parameters of WEDM in NSGA.
The probability of cross enables iterating percentages ranging from $60 \%$ to $100 \%$ of the population may be high such that thus generate new individuals with better characteristics of solution, for this study, a $70 \%$ value for the variable cross took real. Taking into consideration the lack of diversity in the population and the random replacement of individuals the probability of mutation of real variables was estimated at $30 \%$ of the population. The value of the index for cross distribution and mutation of 15 individuals was determined for the first and 30 for the second, there is no binary variables as actual numbers are estimated in the RPD previously.

\subsection{Implement results NSGA-II: Objective functions MRR and Ra}

After running the NSGA-II algorithm, the best values for each variable are collected after 100 generations met the proposed algorithm, running as optimal values shown in the following table 6 .

\begin{tabular}{lll}
\hline Variables & $\begin{array}{l}\text { Objective } \\
\text { functions 1 }\end{array}$ & $\begin{array}{l}\text { Objective } \\
\text { functions 2 }\end{array}$ \\
\hline MRR $\left(\frac{\mathrm{m}^{2}}{h r}\right)$ & 8.86 & 2.57 \\
$\mathrm{Ra}(\mu \mathrm{in})$ & 157.3 & 115.4 \\
$\mathrm{~A}(\mu \mathrm{s})$ & 1.19 & 0.6 \\
$\mathrm{~B}(\mu \mathrm{s})$ & 9.99 & 6.00 \\
Ws $(\mathrm{m} / \mathrm{min})$ & 6.00 & 11.99 \\
Hardness (HRB) & 55.20 & 55.20 \\
\hline
\end{tabular}

Table. 6. Values of the objective functions.

It is noteworthy that the results can be read as follows: for each objective function the maximized or minimized values of each of the two quality characteristics (MRR and Ra) as a side effect are determined magnitudes are also obtained another feature that is not objective; also the four variables are optimized value. The first column is the quality characteristic of MRR which meets the objective to be maximized and the second column contains the minimum value of Ra feature also serving its purpose. After completing the study NSGA implemented to optimize the results, we can draw the thrown values and convert them into optimal levels of 
operation, the results using GA for "Levels selected for MRR” are: Pulse ON-time $(A)=2$, Pulse Off-time $(B)=2$, Wire speed $(\mathrm{Ws})=1$, Noise factor (Hardness)=2. The results for "Levels selected for Ra" are: Pulse ON-time $(\mathrm{A})=1$, Pulse Off-time $(\mathrm{B})=1$, Wire speed (Ws) $=2$, Noise factor (Hardness) $=2$.

This means that even a maximized value of MRR of magnitude $8.86\left(\mathrm{in}^{2} / \mathrm{hr}\right)$ should be chosen operating levels in column 1, to a minimized value of Ra magnitude 115.4 ( $\mu$ in) is will choose the operating levels of the second column.

\subsection{Experimental Confirmation}

Experimental confirmation is the final step in the process of design of experiments in this study in order to validate the results developed during the analysis phase of implementing RPD. Validation of the results obtained after calculating the signal/noise generated operating levels.

\subsection{Calculation of S/N}

The ratio $\mathrm{S} / \mathrm{N}$ is calculated for each design, starting with the original design that sheds the WEDM, then the Design of Experiments based on RPD and finally the de-sign using multiobjective optimization to the implementation of NSGA-II. The S/N ratio is measured in decibels (db) to express variability. The higher the value of the $\mathrm{S} / \mathrm{N}$ ratio, the lower the variability. The following table 7 represents the gain $\mathrm{S} / \mathrm{N}$ against reducing variability.

\begin{tabular}{|c|c|c|c|c|}
\hline $\begin{array}{l}\text { Gain } \\
\text { (db) }\end{array}$ & $\mathrm{S} / \mathrm{N}$ & $\begin{array}{l}\text { Reducing } \\
\text { Variability }\end{array}$ & $\begin{array}{l}\text { Gain } \\
\text { S/N } \\
\text { (db) }\end{array}$ & $\begin{array}{l}\text { Reducing } \\
\text { Variability }\end{array}$ \\
\hline 0.1 & & $1.1 \%$ & 0.2 & $2.3 \%$ \\
\hline 0.5 & & $5.6 \%$ & 1.0 & $10.9 \%$ \\
\hline 1.5 & & $15.9 \%$ & 2.0 & $20.6 \%$ \\
\hline 2.5 & & $25.1 \%$ & 3.0 & $29.3 \%$ \\
\hline 4.0 & & $37.0 \%$ & 5.0 & $43.9 \%$ \\
\hline 6.0 & & $50.0 \%$ & 9.0 & $64.6 \%$ \\
\hline 12 & & $75.0 \%$ & 15 & $82.3 \%$ \\
\hline 18 & & $87.5 \%$ & 21 & $91.2 \%$ \\
\hline 24 & & $93.8 \%$ & & \\
\hline
\end{tabular}

\subsection{Confirmation Test}

Confirmatory testing was performed in 310 Robofil machine which data quality characteristics of the material removal rate (MRR) and surface roughness ( $\mathrm{Ra}$ ) when cutting controlled implementation levels were collected optimal RPD obtained by NSGA-II and the experimental verification and making the $\mathrm{S} / \mathrm{N}$ were obtained with the statistical tool of Minitab 16 and described below, it is worth mentioning that there are two different tests since a confirmation meets the objective of maximize material removal rate (MRR) and the other objective is to minimize the surface roughness (Ra). This reflects the fact that each quality characteristic has its priority as the target. The results for MRR confirmation are: Original Design (WEDM) $=11.3276$, Robust Design $\quad(R P D)=19.1501, \quad$ Multi-objective Optimization (NSGA-II) $=19.1500$. The results for Ra are: Original Desing (WEDM) $=-43.9362$, Robust Design (RPD) $=-41.8796$ and Multiobjective Optimization (NSGA-II)=-41.0954 The S/R for Robust Design (RPD) and multiobjective optimization using NSGA-II, to maximize MRR differs in magnitude, this is because as the two experiments were optimal thereof levels, so the same gain is obtained for both experiments. The Robust Design (RPD) and Multi-Objective Optimization using NSGAII, to minimize $\mathrm{Ra}$ if you have a marked difference, this will be reflected in the profit that has everyone on the original design.

\section{Conclusions}

The selection of the design of experiments (DOE) provided optimal levels WEDM machining with the correct selection and excellent methodology as is Dr. Taguchi [TAGUCHI, G. 1993] RPD generating product quality, and impact on the total cost. Were optimized and confirmed the results with Multiobjective optimization using NSGA. With the interpolation of the values of S/N and Figure 7. The variability is reduced: Robust Design $(\mathrm{RPD})=57 \%$ and Multi-objective Optimization $($ NSGA-II) $=57 \%$. 
For the case, that the process needs a roughing regime maximizing material removal rate, the expected gain with this rugged design and confirmed by NSGA-II is $57 \%$. This results in more than half of the reduced machining time yielding optimal values of operation and minimizes the cost of inputs. Robust Design $(\mathrm{RPD})=21 \%$ and Multi-objective Optimization $($ NSGA-II $)=29 \%$.

In a Multi-objective optimization the process needs a system of fine finish, the surface roughness is minimized by applying robust design (RPD) gain on the original design by machine yielded $21 \%$ is obtained a gain of $29 \%$ on Original Design is obtained, this means that the quality characteristic $\mathrm{Ra}$ is further optimized using GA and NSGA-II specific. These values reflect a decreased tool wear deviations from specifications and will have a greater control of the process. Also by the RPD it was found that the whips interactions are taken into control factors are:
Duration of discharge (pulse on time) "A" and wire feed speed "Ws".

Time between pulses (pulse off time) "B" and wire feed speed "Ws".

GA has the ability to find the global optimal parameters, while traditional optimization techniques such as RPD normally tend to be found in local optima, this check was achieved in the study. Performance measures with the same importance to the quality characteristics and their weights, where the high value and low value MRR for $\mathrm{Ra}$ were equally important objectives in the WEDM process were evaluated.

\section{Acknowledgment}

The authors are extremely thankful to the referees for their valuable comments and suggestions which contributed significantly to improve the quality of this research.

\section{$7 \quad$ References}

[TAGUCHI, G. 1993]

[MAHAPATRA, S. et al. 2006]

[KALYANMOY, D. et al. 2002]

[KUEHL, R. 2001]

[LIAO, Y. et al. 1997]
TAGUCHI, G. Taguchi on robust technology development: Bringing quality engineering upstream, ASME Press, 1993. New York.

MAHAPATRA, S. Optimization of wire electrical discharge machining (WEDM) process parameters using Taguchi method. Journal of Advanced Manufacturing Technology. 2006.

KALYANMOY, D. A Fast and Elitist multi-objective Genetic Algorithm: NGSA-II. IEEE Transactions on Evolutionary Computation (IEEE-TEC). 2002.

KUEHL, R. Diseño de experimentos: Principios estadísticos de diseño y análisis de investigación. International Thomson, 2001. México.

LIAO, Y. A study on the machining-parameters optimization of wire electrical discharge machining. Journal of Materials Processing Technology. 1997.. 
Carlos Alberto Ochoa Ortiz-Zezzatti (B.S. '94, Master '00, PhD. '04, Postdoctoral Researcher,'06, and Industrial Postdoctoral Research '09). He joined the Juarez City University in 2008. He has 7 BOOK, and 17 chapters in books related with AI. He has supervised 37 Ph.D. theses, 47 M.Sc. theses and 49 undergraduate theses. He participated in the organization of COMCEV'07, COMCEV'08, HAIS'07, HAIS'08, HAIS'09, HAIS'10, HAIS'11, HAIS'12, ENC'06, ENC'07, ENC'08 and MICAI'08, MICAI'09, MICAI'10 \& MICAI'11. His research interests include evolutionary computation, natural processing language, anthropometrics characterization and Social Data Mining.

María de Lourdes Yolanda Margain Fuentes has a Bachelor degree in Computer Information Systems from the Autonomous University of Aguascalientes. She studied a Master degree in Computer Science and a PhD in Computer Science at the same university. She is the Director of Postgraduate Studies in Polytechnic University of Aguascalientes. Her research interests include artificial intelligence, software engineering and e-learning.

Francisco Javier Ornelas Zapata has a Bachelor degree in Information Technology from the Technological Institute of Aguascalientes. She studied a Master degree in Computer Science and a PhD in Computer Science at the Autonomous University of Aguascalientes. His research interests include artificial intelligence, evolutionary computation and intelligent tutors.

Sandra Guadalupe Jiménez González has a Bachelor degree in Information Strategic Systems in the Polytechnic University of Aguascalientes. Her study field is most oriented to semantic web and eLearning research.

Teresa Guadalupe Padilla Velázquez has a Bachelor degree in Information Strategic Systems in the Polytechnic University of Aguascalientes. Her research interest include artificial intelligence, ubiquitous computing and decision support systems. 